\title{
Memory Effect in Chemotaxis Equation
}

\author{
Bhupendra Singh1, Loukrakpam Kennedy Meitei2, Ranjit Kumar², Varun Malik², \\ Yogesh Kumar'2, Nihal Kumar² \\ ${ }^{1}$ Department of Physics, Atmaram Sanatan Dharma College, University of Delhi, Delhi, India \\ ${ }^{2}$ Department of Physics, Dyal Singh College, University of Delhi, Delhi, India \\ Email: *kennedy4mangang@gmail.com
}

Received 3 April 2016; accepted 15 June 2016; published 20 June 2016

Copyright (C) 2016 by authors and Scientific Research Publishing Inc.

This work is licensed under the Creative Commons Attribution International License (CC BY). http://creativecommons.org/licenses/by/4.0/

(c) (i) Open Access

\section{Abstract}

Diffusion-Reaction (DR) equation has been used to model a large number of phenomena in nature. It may be mentioned that a linear diffusion equation does not exhibit any traveling wave solution. But there are a vast number of phenomena in different branches not only of science but also of social sciences where diffusion plays an important role and the underlying dynamical system exhibits traveling wave features. In contrast to the simple diffusion when the reaction kinetics is combined with diffusion, traveling waves of chemical concentration are found to exist. This can affect a biochemical change, very much faster than straight diffusional processes. This kind of coupling results into a nonlinear (NL) DR equation. In recent years, memory effect in DR equation has been found to play an important role in many branches of science. The effect of memory enters into the dynamics of NL DR equation through its influence on the speed of the travelling wavefront. In the present work, chemotaxis equation with source term is studied in the presence of finite memory and its solution is compared with the corresponding chemotaxis equation without finite memory. Also, a comparison is made between Fisher-Burger equation and chemotaxis equation in the presence of finite memory. We have shown that nonlinear diffusion-reaction-convection equation is equivalent to chemotaxis equation.

\section{Keywords}

Chemotaxis, Nonlinear Diffusion-Reaction Equation, Finite Memory Effect, Solitary Wave Solutions

\section{Introduction}

Diffusion-Reaction (DR) equation has been used to explain many phenomena in nature [1]. On the other hand, DR equation with finite memory transport has played important role in recent years [2]-[6]. The diffusion

${ }^{*}$ Corresponding author. 
equation with finite memory have been used to explain the forest fire [7], population growth models [8], the effect of legalisation of abortion law on life of women in Italy [9], diffusion of drugs through skin membrane [10].

In addition to diffusion, there are many phenomena in nature in which convection velocity term also becomes important [11]-[13]. Convective velocity is a measure of the bias in the system (think of a dye injected into a running stream). The case when convective velocity became time-dependent was studied by Franosch and Nelson [11]. They studied numerically how this time-dependent convection or the so called "wind" affected the population near a hot spot of favorable growth rates (an oasis) surrounded by a less favorable "desert region". Nonlinear convective velocity term arises in many physical problems including one dimensional turbulence, sound waves in viscous medium, shock waves in viscous medium and so on [14].

Nonlinear convective flux term arises naturally in the study of chemotaxis equation [15]. While diffusion arises due to random motion of the organism, the movement of certain species can be influenced by the presence of chemoattractant (which can generate the directed movement in the population) [1]. For example, insects, single cell organism, bacteria and animals rely on an acute sense of smell for conveying information between members of the species and the chemicals which are involved in this process are called pheromones. Movement of organism in response to environmental agents (chemical stimulus) is called taxis and movement induced by chemical stimulus is referred to as chemotaxis [1] [15]-[19]. Thus chemically directed movement is called chemotaxis.

For example, the female silk moth Bombyx mori exudes a pheromone, called bombykol, as a sex attractant for the male, which has a remarkably efficient antenna filter to measure the bombykol concentration, and it moves in the direction of increasing concentration. The acute sense of smell of many deep sea fish is particularly important for communication and predation [1] [20]. It is not only in animal and insect ecology that the mechanism of chemotaxis is important. It can equally be crucial in biological processes. For example, when a bacterial infection invades the body it may be attacked by movement of cells towards the source as a result of chemotaxis.

It has been shown that certain species of bacteria and insects can move toward higher concentrations of nutrients [1]. Concentration $s(x, t)$ of the substrate or attractant is governed by the equation [17]

$$
\frac{\partial s}{\partial t}=-G(s) u+D_{0} \frac{\partial^{2} s}{\partial x^{2}},
$$

where $G(s)$ is the concentration of the attractant per cell, $u(x, t)$ is the density of the bacteria and $D_{0}$ is the diffusion constant of the attractant. For most of the practical purpose, $G(s)$ is taken as constant and diffusion constant $D_{0}$ is also neglected [21]. Arguments to justify this approximation are given in Ref [21]. Under this assumption Equation (1) reduces to

$$
\frac{\partial s}{\partial t}=-G u \text {. }
$$

On the other hand concentration of the bacteria is described by the equation [16]

$$
\frac{\partial u}{\partial t}=\frac{\partial}{\partial x}\left[\mu(s) \frac{\partial u}{\partial x}\right]-\frac{\partial}{\partial x}\left[u \chi(s) \frac{\partial s}{\partial x}\right]+f(u)
$$

where the first tern on the right side represent the motion of the bacteria in the absence of chemotaxis. In the absence of chemical gradient $\left(\frac{\partial s}{\partial x}=0\right)$, Equation (3) becomes identical to the diffusion-reaction equation in the presence of source term $f(u)$. Here motility factor, $\mu$, takes the place of the diffusion coefficient $D$. In Equation (3) $\mu$ is taken as function of substrate concentration s. In principle $\mu$ could also vary with bacterial concentration $u$ and space variable $x$. But the effect of substrate concentration is not known at present [16]. For most of the practical purpose $\mu$ is taken as constant.

The second tern on the right side of Equation (3) describes the chemotactic response of the species. In Equation (3), $u \chi(s)\left[\frac{\partial s}{\partial x}\right]$ is flux of species due to chemotaxis where $\chi(s)$ is a measure of strength of chemotaxis, and is termed as chemotactic coefficient. The function $\chi$ is also called the chemotactic sensitivity 
function. In the next section we will discuss memory effect in DR equation.

\section{Memory Effect in DR Equation}

Memory effect in DR equation arises when dispersal of the particle is not mutually independent [2]-[6]. In particular, note that the solution of the one dimensional diffusion equation (the boundary of the problem is at infinity, i.e., $-\infty<x<\infty$ )

$$
\frac{\partial u}{\partial t}=D \frac{\partial^{2} u}{\partial x^{2}}
$$

is given by

$$
u(t, x)=\frac{1}{\sqrt{4 D \pi t}} \exp \left(-\frac{x^{2}}{4 D t}\right)
$$

At $t=0$, the solution of the equation is Dirac delta function, i.e.,

$$
u(x, t=0)=\delta(x)
$$

Thus, at $t=0$ all the particles are sitting at $x=0$. For $t>0$, the solution of the equation is non zero for all $x$. If we take a value of $x$ such that $|x|>c t$, where $c$ is the speed of light, we see that there is a finite probability, however small, for particles to diffuse at superluminal speeds. The error lies in the diffusion equation itself, which does not recognize any limiting propagation speed. Thus it becomes necessary to include memory effect, which takes care of the finite speed. When memory effect is taken into account then we have the following modification of Fick's law [2] in the presence of nonlinear convection term

$$
\begin{gathered}
J(x, t+\tau)=-D \frac{\partial u}{\partial x}+g(u) v, \\
\frac{\partial u}{\partial t}=-\frac{\partial J}{\partial x}+f(u),
\end{gathered}
$$

where $u=u(x, t)$, is the concentration or the density variable depending on the phenomenon under study; $v$ is the coefficient of nonlinear convective flux term $g(u)$ and $D$ is the diffusion coefficient. Here $\frac{\partial u}{\partial t}$ is the time rate of change of concentration at time $t$ and $J(x, t+\tau)$ is the flux at a later time $t+\tau$, while $f(u)$ is the source term. Here $\tau$ is delay time and its value depends on the system under study [9] [22] [23]. For experimental determination of $\tau$, see ref. [22] [23]. After simplifying Equations (4) and (5) we get

$$
u_{t t}-\beta D u_{x x}-f^{\prime}(u) u_{t}+\beta\left(u_{t}-f(u)\right)+\beta v g^{\prime}(u) u_{x}=0 .
$$

Here $\beta \equiv \frac{1}{\tau}, \quad f^{\prime}(u)=\frac{\mathrm{d} f}{\mathrm{~d} u}$ and $g^{\prime}(u)=\frac{\mathrm{d} g}{\mathrm{~d} u}$. In particular for $f(u)=\alpha u-\gamma u^{2}$ and $g(u)=u^{2}$, Equation (6) reduces to Fisher-Burger equation with finite memory [6]

$$
u_{t t}-\beta D u_{x x}-f^{\prime}(u) u_{t}+\beta\left(u_{t}-f(u)\right)+k \beta u u_{x}=0
$$

where $k \equiv 2 v$. Note that Equation (6) describe a transport phenomenon in which both diffusion and convection processes are of equal importance. After using the transformation $\xi=x-w t$ in Equation (6), we get

$$
\left(w^{2}-\beta D\right) u^{\prime \prime}+\left(f^{\prime}(u)-\beta\right) w u^{\prime}-\beta f(u)+\beta v g^{\prime}(u) u^{\prime}=0 .
$$

By taking $\tau=1 / \beta=0$ in Equations (6) and (7) one obtain the corresponding DR equation without finite memory transport

$$
u_{t}+v g^{\prime}(u) u_{x}=D u_{x x}+f(u)
$$

and

$$
D u^{\prime \prime}+w u^{\prime}-v g^{\prime}(u) u^{\prime}+f(u)=0 .
$$


One can see from above that Equations (6) and (7) are hyperbolic nonlinear DR equation while Equations (8) and (9) are parabolic nonlinear DR equation.

\section{Memory Effect in Chemotaxis Equation}

In the presence of finite memory, Equation (5) remains unchanged while Equation (4) gets modified to

$$
J(x, t+\tau)=-\left[\mu(s) \frac{\partial u}{\partial x}-u \chi(s) \frac{\partial s}{\partial x}\right] .
$$

Simplifying Equations (5) and (10) one obtains

$$
u_{t t}+\left(\beta-f^{\prime}(u)\right) u_{t}-\beta f(u)-\beta \frac{\partial}{\partial x}\left[\mu(s) \frac{\partial u}{\partial x}\right]+\beta \frac{\partial}{\partial x}\left[u \chi(s) \frac{\partial s}{\partial x}\right]=0
$$

In Equation (11) we will take $\mu(s)=\mu_{0}, \quad \chi(s)=\chi_{0}$. Afetr this substitution Equation (11) becomes

$$
u_{t t}+\left(\beta-f^{\prime}(u)\right) u_{t}-\beta f(u)-\beta \mu_{0} u_{x x}+\beta \chi_{0} s_{x} u_{x}+\beta \chi_{0} u s_{x x}=0 .
$$

Now using the transformation $\xi=x-w t$ in Equations (2) and (12) we get

$$
\begin{gathered}
s^{\prime}=\frac{G}{w} u, \\
\left(w^{2}-\beta \mu_{0}\right) u^{\prime \prime}+\left(f^{\prime}(u)-\beta\right) w u^{\prime}-\beta f(u)+\beta \chi_{0} s^{\prime} u^{\prime}+\beta \chi_{0} u s^{\prime \prime}=0 .
\end{gathered}
$$

For $\tau=1 / \beta=0$, Equations (12) and (14) becomes

$$
\begin{gathered}
u_{t}-\mu_{0} u_{x x}+\chi_{0} s_{x} u_{x}+\chi_{0} u s_{x x}=f(u), \\
\mu_{0} u^{\prime \prime}+w u^{\prime}-\chi_{0} s^{\prime} u^{\prime}-\chi_{0} u s^{\prime \prime}+f(u)=0 .
\end{gathered}
$$

From Equation (13) one can see that $s^{\prime \prime}=G u^{\prime} / w$. Substituting the value of $s^{\prime}$ and $s^{\prime \prime}$ from Equation (13) into Equation (14) one obtains

$$
\left(w^{2}-\beta \mu_{0}\right) u^{\prime \prime}+\left(f^{\prime}(u)-\beta\right) w u^{\prime}-\beta f(u)+\frac{2 \beta \chi_{0} G}{w} u u^{\prime}=0 .
$$

In Equation (7) if we take $g(u)=u^{2}$, then we obtain the following equation

$$
\left(w^{2}-\beta D\right) u^{\prime \prime}+\left(f^{\prime}(u)-\beta\right) w u^{\prime}-\beta f(u)+2 \beta v u u^{\prime}=0 .
$$

Now comparing Equations (17) and (18) one can see that $D=\mu_{0}$ and $v=\chi_{0} G / w$. Under this condition Equations (7) and (17) becomes identical. Note that here $v$ is coefficient of nonlinear convection term and from Equation (17) one can see that this coefficient depends on $\chi_{0}, G$ and wave velocity $w$. Thus, we have mapped the chemotaxis equation to nonlinear diffusion-reaction-convection equation. In this mapping we have assumed that $v=\frac{\chi_{0} G}{w}$ and hence by measuring $\chi_{0}, G$ and $w$, experimentally, we can find the velue of convective velocity term $v$. Similarly, by measuring $\mu_{0}$ experimentally, we can determine the diffusion coefficient $D$.

\section{Solutions}

For Fisher type reaction term $f(u)=\alpha u-\gamma u^{2}$, Equation (17) or (18) takes the form

$$
\left(w^{2}-\beta D\right) u^{\prime \prime}+w(\alpha-\beta) u^{\prime}+(k \beta-2 \gamma w) u u^{\prime}-\beta \alpha u+\beta \gamma u^{2}=0,
$$

where $k \equiv 2 v=\frac{2 \chi_{0} G}{w}$. By taking $\tau=1 / \beta=0$ in Equation (19) one obtain the corresponding nonlinear DR equation without finite memory transport

$$
D u^{\prime \prime}+w u^{\prime}-k u u^{\prime}+\alpha u-\gamma u^{2}=0 .
$$


Solutions of Equations (19) and (20) is already obtained by us in ref. [6]. Here we will write the solution of Equation (19) as

$$
u(\xi)=\frac{\alpha}{2 \gamma}\left[1-\tanh \left(\frac{\gamma k \alpha(\alpha+\beta)}{\left(4 D \beta \gamma^{2}-\alpha^{2} k^{2}\right)} \xi\right)\right]
$$

and

$$
u(\xi)=\frac{\alpha}{2 \gamma}\left[1-\operatorname{coth}\left(\frac{\gamma k \alpha(\alpha+\beta)}{\left(4 D \beta \gamma^{2}-\alpha^{2} k^{2}\right)} \xi\right)\right],
$$

where wave velocity $w$ is given by

$$
w=\frac{\beta\left(\alpha k^{2}+4 D \gamma^{2}\right)}{2 \gamma k(\alpha+\beta)} .
$$

Equation (21) is a solitary wave solution of Equation (19) whereas solution (22) diverges. Since $u(\xi)$ represent the concentration of certain species which cannot go to infinity hence solution (22) is physically not acceptable. On the other hand solutions of Equation (20) is given by

$$
u(\xi)=\frac{\alpha}{2 \gamma}\left[1-\tanh \left(\frac{\alpha k}{4 \gamma D} \xi\right)\right]
$$

and

$$
u(\xi)=\frac{\alpha}{2 \gamma}\left[1-\operatorname{coth}\left(\frac{\alpha k}{4 \gamma D} \xi\right)\right]
$$

and wave speed $w$ as

$$
w=\frac{\alpha k^{2}+4 D \gamma^{2}}{2 \gamma k} .
$$

Equation (24) is again a solitary wave solution of Equation (20) while Equation (25) is physically not acceptable. Now using Equation (13), we have

$$
s(\xi)=\int \frac{G}{w} u(\xi) \mathrm{d} \xi+C,
$$

where $C$ is constant of integration. Substituting the value of $u(\xi)$ from Equations (21) and (22) in Equation (27), we obtain the following value of $s(\xi)$ of Equation (13) as

$$
s(\xi)=\frac{G \alpha}{2 \gamma w}\left[\xi-\frac{\left(4 D \beta \gamma^{2}-\alpha^{2} k^{2}\right)}{\gamma k \alpha(\alpha+\beta)} \ln \left[\cosh \left(\frac{\gamma k \alpha(\alpha+\beta)}{\left(4 D \beta \gamma^{2}-\alpha^{2} k^{2}\right)} \xi\right)\right]\right]+C,
$$

and

$$
s(\xi)=\frac{G \alpha}{2 \gamma w}\left[\xi-\frac{\left(4 D \beta \gamma^{2}-\alpha^{2} k^{2}\right)}{\gamma k \alpha(\alpha+\beta)} \ln \left[\sinh \left(\frac{\gamma k \alpha(\alpha+\beta)}{\left(4 D \beta \gamma^{2}-\alpha^{2} k^{2}\right)} \xi\right)\right]\right]+C,
$$

where $w$ is given by Equation (23). Similiarly, substituting the value of $u(\xi)$ from Equations (24) and (25) in Equation (27), we obtain the following value of $s(\xi)$ of Equation (13) as

$$
s(\xi)=\frac{G \alpha}{2 \gamma w}\left[\xi-\frac{4 \gamma D}{\alpha k} \ln \left[\cosh \left(\frac{\alpha k}{4 \gamma D} \xi\right)\right]\right]+C,
$$

and 


$$
s(\xi)=\frac{G \alpha}{2 \gamma w}\left[\xi-\frac{4 \gamma D}{\alpha k} \ln \left[\sinh \left(\frac{\alpha k}{4 \gamma D} \xi\right)\right]\right]+C,
$$

where $w$ is given by Equation (26). From Equations (28)-(31) one can see that concentration $s(x, t)$ of attractant increases with $G$ and decreases as wave speed $w$ increases. Also, it is directly proportional to coefficient of linear term $\alpha$ and inversely proportional to coefficient of nonlinear term $\gamma$.

\section{Concluding Remarks}

Certain aspects ignored earlier [10] in the studies of the chemotaxis equation involving quadratic nonlinearity are now investigated in this work. We have studied the chemotaxis equation with the source term and finite memory transport in particular. The existence of solitary wave solutions of Equations (19) and (20) is demonstrated explicitly. We have also obtained an exact solutions for concentration $s(x, t)$. Also, a correspondence between nonlinear Diffusion-Reaction-Convection and chemotaxis equation is established. It is shown that nonlinear DR equation with nonlinear convection term is equivalent to chemotaxis equation. Thus, nonlinear convection terms arise naturally in the study of chemotaxis [10]. Also, from Equation (17) one can see that coefficient of non-linear convective flux, $v=\frac{\chi_{0} G}{w}$, decreases as wave speed $w$ increases. Thus, using the nonlinear Diffusion-Reaction-Convection equation one can find the coefficient of nonlinear convective flux $v$, of chemotaxis equation.

Although result obtained in this paper is highly simplified, the solutions obtained here can explain such physical phenomena which is governed by chemotaxis equation. The case when $\mu$ and $\chi$ depend on bacteria concentration $u$ and space variable $x$ will be discussed in a separate paper. Such studies are in progress.

\section{Acknowledgements}

We would like to thank R. S. Kaushal, Awadhesh Prasad and Ram Ramaswamy for helpful discussion. We would also like to thank Dyal Singh College for providing us the computational facility during the course of this work.

\section{References}

[1] Murray, J.D. (1993) Mathematical Biology. Springer-Verlag, New York.

[2] Cattaneo, C. (1958) Comptes Rendus de l'Académie des Sciences, 247, 431-433.

[3] Abramson, G., Bishop, A.R. and Kenkre, V.M. (2001) Physical Review E, 64, 066615.

[4] Kar, S., Banik, S.K. and Ray, D.S. (2002) Physical Review E, 65, 061909. http://dx.doi.org/10.1103/PhysRevE.65.061909

[5] Kar, S., Banik, S.K. and Ray, D.S. (2003) Journal of Physics A: Mathematical and General, 36, 2771. http://dx.doi.org/10.1088/0305-4470/36/11/308

[6] Mishra, A. and Kumar, R. (2012) Physical Review A, 376, 1833.

[7] Méndez, V. and Llebot, J.E. (1997) Physical Review E, 56, 6557. http://dx.doi.org/10.1103/PhysRevE.56.6557

[8] Méndez, V. andCamacho, J. (1997) Physical Review E, 55, 6476. http://dx.doi.org/10.1103/PhysRevE.55.6476

[9] Caputo, M. and Gloria-Bottini, F. (2011) Natural Science, 3, 694-701. http://dx.doi.org/10.4236/ns.2011.38093

[10] Caputo, M. and Cametti, C. (2009) Journal of Physics D: Applied Physics, 42, 125505. http://dx.doi.org/10.1088/0022-3727/42/12/125505

[11] Franosch, T. and Nelson, D.R. (2000) Journal of Statistical Physics, 99, 1021-1030. http://dx.doi.org/10.1023/A:1018607932123

[12] Mishra, A. and Kumar, R. (2010) Physics Letters A, 374, 2921-2924. http://dx.doi.org/10.1016/j.physleta.2010.03.039

[13] Gilding, B.H. and Kersner, R. (2004) Traveling Waves in Nonlinear Diffusion-Convection Reaction. Birkhäuser Verlag, Basel. http://dx.doi.org/10.1007/978-3-0348-7964-4

[14] Debnath, L. (1997) Nonlinear Partial Differential Equations for Scientists and Engineers. Birkhäuser, Boston. http://dx.doi.org/10.1007/978-1-4899-2846-7 
[15] Benguria, R.D., Depassier, M.C. and Méndez, V. (2004) Physical Review E, 69, 031106. http://dx.doi.org/10.1103/PhysRevE.69.031106

[16] Keller, E.F. and Segel, L.A. (1971) Journal of Theoretical Biology, 30, 235-248. http://dx.doi.org/10.1016/0022-5193(71)90051-8

[17] Rosen, G. (1976) Journal of Theoretical Biology, 59, 371-380. http://dx.doi.org/10.1016/0022-5193(76)90177-6

[18] Sherratt, J.A. (1994) Bulletin of Mathematical Biology, 56, 129-146. http://dx.doi.org/10.1007/BF02458292

[19] Adler, J. and Dahl, M. (1967) Microbiology, 46, 161-173. http://dx.doi.org/10.1099/00221287-46-2-161

[20] Di Francesco, M. Mathematical Models in Life Sciences. Lecture Notes.

[21] Segel, L.A. (1972) Lecture Notes on Mathematics in the Life Sciences, Vol. 4. American Mathematical Society, Providence, 1-47.

[22] Aoki, K. and Xiang, C.Y. (2007) The Journal of Physical Chemistry C, 111, 15433-15439. http://dx.doi.org/10.1021/jp071757g

[23] Iaffaldano, G., Caputo, M. and Martino, S. (2006) Hydrology and Earth System Sciences, 10, 93-100. http://dx.doi.org/10.5194/hess-10-93-2006 